\section{Elimination of a specific histone H3K14 acetyltransferase complex bypasses the RNAi pathway to regulate pericentric heterochromatin functions}

\author{
Bharat D. Reddy, ${ }^{1}$ Yu Wang, ${ }_{1}^{1}$ Lifang Niu, ${ }^{1}$ \\ Emily C. Higuchi, ${ }^{2}$ Samuel B. Marguerat, ${ }^{3}$ \\ Jürg Bähler, ${ }^{3}$ Gerald R. Smith, ${ }^{2}$ \\ and Songtao $\mathrm{Jia}^{1,4}$
}

${ }^{1}$ Department of Biological Sciences, Columbia University, New York, New York 10027, USA; ${ }^{2}$ Division of Basic Sciences, Fred Hutchinson Cancer Research Center, Seattle, Washington 98109, USA; ${ }^{3}$ Department of Genetics, Evolution, and Environment, UCL Cancer Institute, University College London, London WC1E 6BT, United Kingdom

In Schizosaccharomyces pombe, the RNAi pathway is required for the formation of pericentric heterochromatin, proper chromosome segregation, and repression of pericentric meiotic recombination. Here we demonstrate that, when the activity of the histone H3 Lys 14 (H3K14) acetyltransferase Mst2 is eliminated, the RNAi machinery is no longer required for pericentric heterochromatin functions. We further reveal that reducing RNA polymerase II recruitment to pericentric regions is essential for maintaining heterochromatin in the absence of RNAi.

Supplemental material is available for this article.

Received September 15, 2010; revised version accepted December 13, 2010.

Repetitive DNA elements are major components of most eukaryotic genomes and are preferential sites for the assembly of heterochromatin structures (Grewal and Jia 2007; Peng and Karpen 2008). Heterochromatin recruits a myriad of proteins to control diverse processes such as transcription, recombination, and chromosome segregation (Grewal and Jia 2007). The formation of heterochromatin requires the concerted actions of several histone-modifying enzymes, which lead to methylation of histone H3 Lys 9 (H3K9me) and the subsequent recruitment of structural proteins such as HP1 (Grewal and Jia 2007). Heterochromatin assembly also depends on the RNAi pathway, which targets histone-modifying activities to repeat regions (Matzke and Birchler 2005; Buhler and Moazed 2007; Grewal and Jia 2007).

Extensively characterized in fission yeast (Supplemental Fig. S1; Buhler and Moazed 2007; Grewal and Jia 2007),

[Keywords: RNAi; heterochromatin; H3K14 acetylation; Mst2; transcription; meiotic recombination]

${ }^{4}$ Corresponding author.

E-MAIL sj2274@columbia.edu, FAX (212) 865-8246.

Article is online at http://www.genesdev.org/cgi/doi/10.1101/gad.1993611.
RNAi-mediated heterochromatin assembly is triggered by the generation of dsRNAs from repetitive DNA elements, which are then processed by the Dicer nuclease into siRNAs. The siRNAs are loaded onto the ARC complex (composed of the Argonaute protein Agol plus Arb1 and Arb2) and then the RITS complex (composed of Ago1, a chromodomain protein Chp1, and a GW domain protein, Tas3) and guide RITS to nascent transcripts originating from DNA repeats. RITS recruits the histone methyltransferase complex CLRC (composed of H3K9 methyltransferase Clr4, E3 ubiquitin ligase Cul4, Rik1, Raf1, and Raf2) via Stcl to initiate H3K9me (Bayne et al. 2010), which subsequently recruits the HP1 orthologs Swi6 and Chp2. RITS also recognizes $\mathrm{H} 3 \mathrm{~K} 9 \mathrm{me}$ via $\mathrm{Chp} 1$ and recruits the RDRC complex (composed of an RNA-dependent RNA polymerase [Rdp1], a putative helicase [Hrr1], and a polyA polymerase [Cid12]). RDRC promotes the production of dsRNAs, resulting in a positive feedback loop that strengthens heterochromatin structures.

In fission yeast, the pericentric regions, subtelomeres, and silent mating type region are major sites of heterochromatin. All of these regions contain repetitive DNA elements of a common origin and require functional RNAi for heterochromatin establishment (Grewal and Jia 2007). However, at the silent mating type region and subtelomeres, RNAi is not necessary for the subsequent maintenance of heterochromatin due to the presence of redundant RNAi-independent heterochromatin assembly pathways (Grewal and Jia 2007). Interestingly, heterochromatin maintenance at pericentric regions is RNAi-dependent, although the mechanism of this maintenance is not clear (Sadaie et al. 2004). Consequently, the loss of RNAi components selectively disrupts pericentric heterochromatin, making this region ideal for studying the mechanism of RNAi-mediated heterochromatin assembly (Buhler and Moazed 2007; Grewal and Jia 2007). Here we show that RNAi is dispensable for pericentric heterochromatin maintenance when a key histone-modifying enzyme, Mst2, is absent. Our results further indicate that locally limiting RNA polymerase II (Pol II) transcription is critical for heterochromatin maintenance in the absence of RNAi.

\section{Results and Discussion}

We recently identified a histone H3K14 acetyltransferase complex containing the MYST family protein Mst2 as the catalytic subunit (Y Wang and S Jia, unpubl.). Loss of Mst2 mildly strengthens silencing near telomeres, and mst24 cells are sensitive to Swi6 overexpression (Gomez et al. 2005), indicating an important yet functionally unclear role for Mst2 in heterochromatin assembly. Since the loss of many heterochromatin components results in increased $\mathrm{H} 3 \mathrm{~K} 14$ acetylation $(\mathrm{H} 3 \mathrm{~K} 14 \mathrm{ac})$ levels at pericentric regions (Sugiyama et al. 2007; Motamedi et al. 2008), we explored whether the loss of Mst2 could bypass the requirement of any components essential for heterochromatin function by examining the expression of a reporter gene inserted at two sites within the pericentric heterochromatin region of chromosome I (otr::ura4 ${ }^{+}$and imr::ura4 ${ }^{+}$) (Fig. 1A; Allshire et al. 1995). The silencing of $\mathrm{ura}^{+}$expression by heterochromatin enables cell growth on media containing 5-fluoroorotic acid (FOA), which is toxic to cells 
A

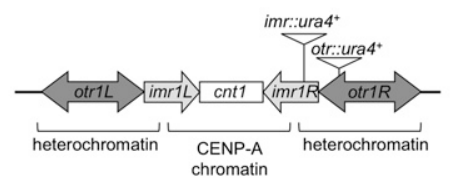

B

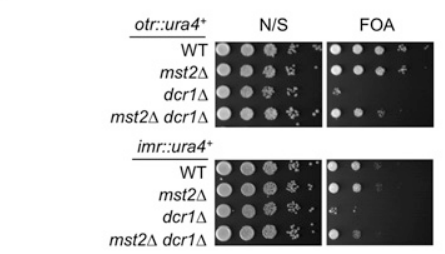

c

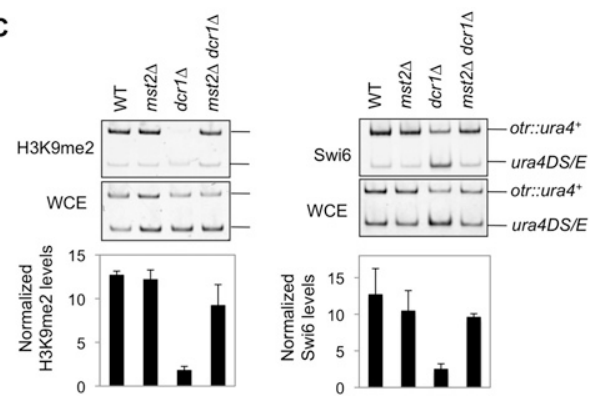

Figure 1. In mst2 $2 \Delta$ cells, the RNAi machinery is no longer required to stabilize pericentric heterochromatin. (A) A schematic diagram of the centromere of chromosome I and the otr::ura $4^{+}$and imr::ura $4^{+}$ reporter genes. $(B)$ Tenfold serial dilution analyses of indicated yeast strains were grown on media with or without FOA to measure the expression of $\mathrm{ura}^{+}$. (N/S) Nonselective medium. (C) Chromatin immunoprecipitation (ChIP) analysis of Swi6 and H3K9me levels at otr::ura4 ${ }^{+}$. DNA immunoprecipitated with Swi6 or H3K9me2 antibodies and from whole-cell extract (WCE) was analyzed by competitive PCR. Fold enrichment is indicated in the graph below. The numbers are averages of three biological repeats. Error bars represent standard deviation.

expressing ura4 ${ }^{+}$. The loss of RNAi components such as Dicer $(d c r 1 \Delta)$ leads to defective heterochromatin assembly and a loss of silencing at these reporters (Fig. 1B). Interestingly, in mst2 $\Delta d c r 1 \Delta$ cells, the silencing of reporter genes is significantly rescued (Fig. 1B) and heterochromatin hallmarks such as H3K9me and Swi6 are considerably restored at pericentric regions (Fig. 1C).

Pericentric heterochromatin is essential for the recruitment of cohesin proteins to ensure proper chromosome segregation during mitosis (for review, see Grewal and Jia 2007). In RNAi mutants such as $d c r 1 \Delta$, cells lose pericentric heterochromatin and exhibit a variety of defects in chromosome segregation, including an increased incidence of lagging chromosomes, a high loss rate of a nonessential minichromosome, and an increased sensitivity to the microtubule-destabilizing agent thiabendazole (TBZ) (Fig. 2A-C; Hall et al. 2003; Volpe et al. 2003). All of these defects are largely rescued in mst $2 \Delta$ dcr $1 \Delta$ cells, suggesting that heterochromatin formed in mst2 $d$ cr $1 \Delta$ cells functions normally.

RNAi is also required for repression of meiotic recombination around centromeres (Ellermeier et al. 2010). In wild-type cells, there is essentially no meiotic recombination between two markers flanking the centromere of chromosome III and $\sim 125 \mathrm{~kb}$ apart (Fig. 2D; Ellermeier et al. 2010). The recombination rate in this region is elevated $\sim 100$-fold in the absence of RNAi (Fig. 2E;
Ellermeier et al. 2010). Importantly, however, this high recombination rate is strongly reduced in mst2 $\Delta$ dcr1s cells. The level of pericentric, meiosis-specific DNA doublestrand breaks (DSBs), which are essential for initiating homologous recombination, is very low in wild-type cells, but is significantly elevated in $d c r 1 \Delta$ cells (Fig. 2F; Supplemental Fig. S2; Ellermeier et al. 2010). However, in mst2s $d c r 1 \Delta$ cells, the level of such DSBs is reduced to nearly wild-type levels, demonstrating that the heterochromatin formed in mst2 $\Delta$ dcr1s cells is capable of inhibiting the formation of meiosis-specific DSBs. Thus, all functions of heterochromatin examined-reduction of gene expression, faithful segregation of chromosomes, and repression of meiotic recombination-are restored by the loss of Mst2 in the absence of RNAi.

Testing the generality of this suppression, we found that mst2A suppressed the silencing defects and TBZ sensitivity of all RNAi mutants examined, such as those in ARC (ago1s, arb1s, and arb2s), RITS (ago1s and
A

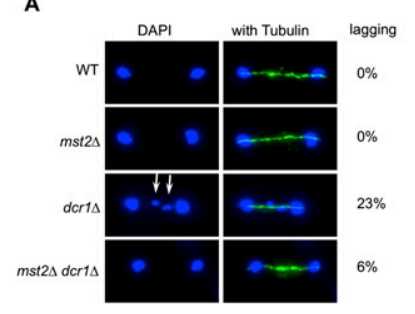

B
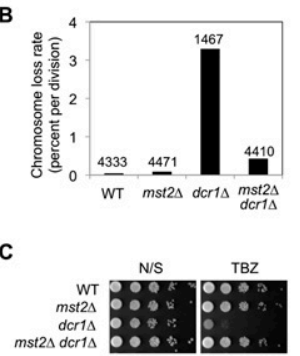

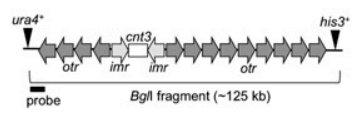

$\mathbf{E}$

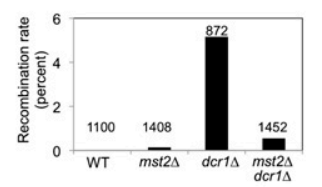

$\mathbf{F}$

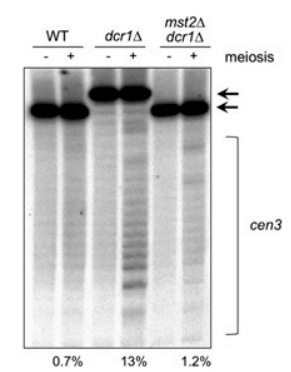

Figure 2. Loss of Mst2 bypasses the requirement of the RNAi machinery for pericentric heterochromatin functions. (A) Cells growing exponentially were stained with DAPI to visualize DNA and with a TAT1 antibody to visualize tubulin. The percentages of cells with lagging chromosomes (indicated by arrows) at late anaphase were determined microscopically $(n=100)$. (B) The loss rate of a nonessential minichromosome, Ch16, was measured. The total number of colonies counted is indicated at the top of each column. (C) Tenfold serial dilution plating assays were performed to measure the sensitivity of cells to TBZ. (D) Schematic diagram of the construct used to measure meiotic recombination across the centromere of chromosome III. The $\mathrm{ura}^{+}$and his $3^{+}$genes were inserted into the $\operatorname{chk} 1^{+}$and near the mid1 $1^{+}$loci, respectively (Ellermeier et al. 2010). (E) The rate of recombination was measured as the percentage of spores that showed nonparental segregation of the $\mathrm{ura}^{+}$and his $3^{+}$ markers. The total number of colonies counted is indicated at the top of each column. $(F)$ Cells were induced for meiosis and harvested at $0 \mathrm{~h}(-)$ and $5 \mathrm{~h}(+)$. DNA was extracted, digested with BglI, and analyzed for DSBs by Southern blot hybridization using a probe, as indicated in $D$. Arrows indicate the unbroken BglI fragment $\mid \sim 125$ $\mathrm{kb})$. The size difference of this fragment in $d c r 1 \Delta$ cells may reflect recombination, meiotic or mitotic, between the outermost repeats (Ellermeier et al. 2010). Meiosis-specific DNA fragments (bracket) result from Rec12-dependent DNA breakage at specific sites around cen3 (Ellermeier et al. 2010). Numbers below the gel are the percent of total DNA broken at $5 \mathrm{~h}$ minus that at $0 \mathrm{~h}$. 
chp1s), and RDRC ( $r d p 1 \Delta$ and cid12 $\Delta$ ), and the recently identified RNAi factors rsh1s (Roguev et al. 2008) and stc1s (Fig. 3A; Supplemental Fig. S3; Bayne et al. 2010). However, mst2 $\Delta$ had no effect in mutants lacking heterochromatin components involved in histone modifications or their recognition, such as those in the CLRC (clr4 $\Delta$, rik1s, raf1s, and raf2 $\Delta$ ), HP1 homologs (swi6s and chp2s), the SHREC complex (clr3s and mit1 $\Delta$ ) (Sugiyama et al. 2007), or histone deacetylase sir2 $\Delta$ (Fig. 3B). These results suggest that Mst2 specifically affects heterochromatin assembly mediated by RNAi.
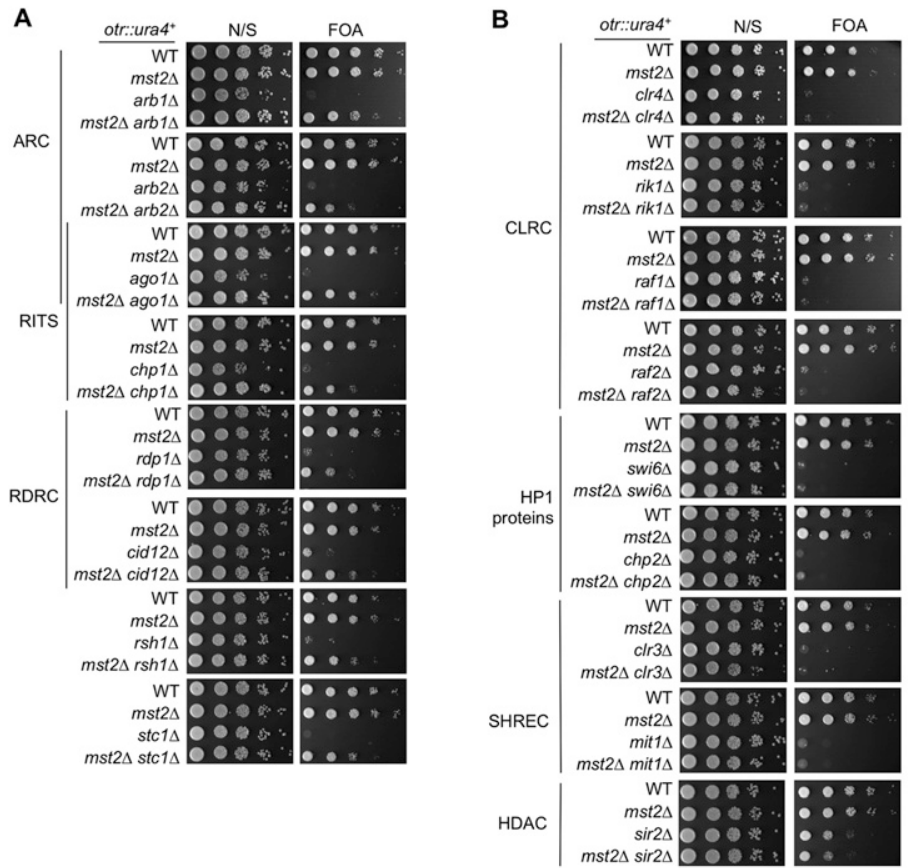

C

D

Grow in the presence of TSA for $30 \mathrm{hrs}$ $\downarrow$

Grow in the absence of TSA for $20 \mathrm{hrs}$
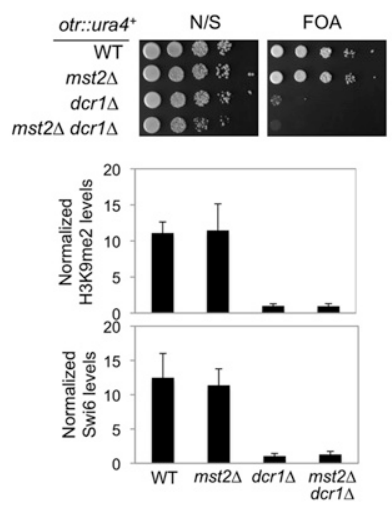

RNAi is required for both the establishment and maintenance of heterochromatin at pericentric regions (Sadaie et al. 2004). To distinguish whether mst2A dcr1s rescues heterochromatin establishment or maintenance, we pulse-treated cells with the histone deacetylase inhibitor trichostatin A (TSA) to erase pre-existing heterochromatin structures (Fig. 3C; Ekwall et al. 1997; Jia et al. 2004). We then examined the re-establishment of heterochromatin as cells recovered. In both $d c r 1 \Delta$ and mst2s dcr1s cells, silencing at otr::ura4 ${ }^{+}$was not re-established, and $\mathrm{H} 3 \mathrm{~K} 9 \mathrm{me} 2$ and Swi6 remained delocalized from pericentric regions (Fig. 3C). To further examine the effect of mst2s dcr1s on heterochromatin establishment, we introduced $c l r 4^{+}$into a mst2s dcr1s clr4s otr::ura4 ${ }^{+}$strain by a genetic cross (Fig. 3D; Hall et al. 2002; Bayne et al. 2010). The

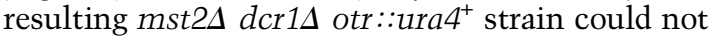
re-establish silencing, at least within the time between spore germination and the assay, as indicated by the loss of growth on FOA media as well as diminished levels of H3K9me and Swi6 at pericentric regions (Fig. 3D). Thus, loss of Mst2 bypasses the requirement of the RNAi pathway for maintaining, but not for establishing, pericentric heterochromatin.

Because the Mst2 complex is a specific histone H3K14 acetyltransferase (Y Wang and S Jia, unpubl.), we next examined whether the enzymatic activity of Mst2 is required to bypass RNAi defects. We found that the catalytically inactive mutant of Mst2 (E274Q) and null mutants of Mst2 complex components essential for its activity, such as Nto1 and Ptf2 (Y Wang and S Jia, unpubl.), also suppress RNAi mutant phenotypes in transcriptional silencing and TBZ sensitivity (Fig. 4A). In contrast, null mutations of two Mst2 complex components not required for acetyltransferase activity (Ptf1 and Eaf6) failed to suppress RNAi defects (data not shown).

Since H3K14ac is correlated with gene transcription in diverse organisms (Pokholok et al. 2005; Wang et al. 2008), we performed microarray analysis to examine whether the ability of mst2s $d c r 1 \Delta$ cells to maintain heterochromatin is the result of altered expression of genes encoding RNAi and heterochromatin components. However, the expression profiles of such genes were not significantly altered (Supplemental Table S1). In addition, we found that siRNAs are absent in mst2 $\Delta$ dcr1s cells, indicating that the rescue of silencing is not a result of activating alternative small RNAproducing pathways (Supplemental Fig. S4).

We hypothesized that the Mst 2 complex directly acetylates $\mathrm{H} 3 \mathrm{~K} 14$ at pericentric regions in the absence of RNAi; i.e., that H3K14 acetylated by Mst2 acts in cis at pericentric regions. As predicted, we found that $\mathrm{H} 3 \mathrm{~K} 14 \mathrm{ac}$ levels at pericentric regions are greatly reduced in $m s t 2 \Delta d c r 1 \Delta$ cells as compared with dcr1s cells (Fig. 4B). Also consistent with our hypothesis, the Mst2 complex component Ntol is enriched at pericentric regions in dcr1s cells (Supplemental Fig. S5A). Since the purified Mst2 complex is capable of acetylating H3K14 irrespective of H3K9me status in vitro (Supplemental Fig. S5B), our results suggest that 
A

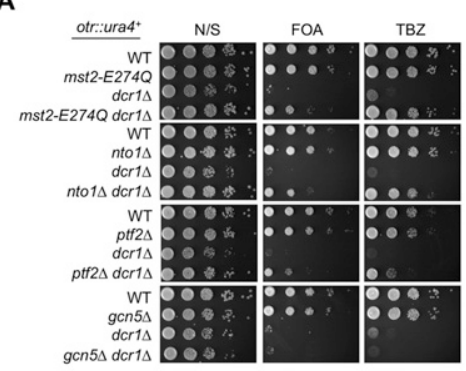

C

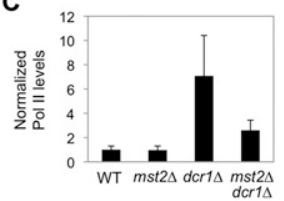

D

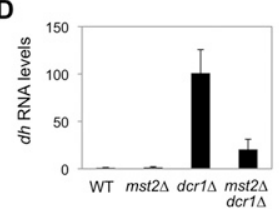

B
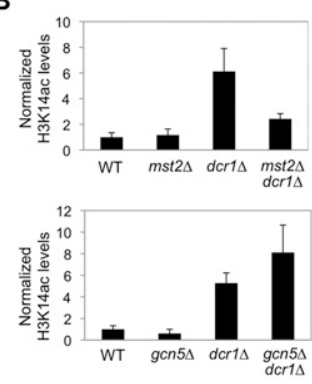

E

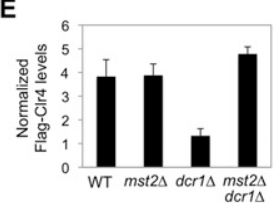

Figure 4. Loss of the Mst2 complex reduces transcription at pericentric heterochromatin in the absence of RNAi. $(A)$ Tenfold serial dilutions of the indicated yeast strains were spotted onto the indicated media to measure the expression of otr::ura4 ${ }^{+}$and the sensitivity to TBZ. (B) ChIP analysis of H3K14 acetylation levels at otr::ura4 ${ }^{+}$. $(C)$ ChIP analysis of Pol II levels at otr::ura $4^{+}$. $(D)$ Real-time RT-PCR analysis of centromeric $d h$ transcript levels. (E) ChIP analysis of Flag-Clr4 levels at otr::ura4 ${ }^{+}$.

RNAi functions to exclude Mst2 from heterochromatin regions in wild-type cells rather than regulate its activity.

Mst2 functions redundantly with another histone acetyltransferase, Gcn5, to regulate global levels of H3K14ac (Nugent et al. 2010; Y Wang and S Jia, unpubl.). However, the loss of Gen5 did not suppress RNAi defects in either transcriptional silencing, TBZ sensitivity, or repression of meiotic recombination (Fig. 4A; Supplemental Fig. S6), and pericentric $\mathrm{H} 3 \mathrm{~K} 14 \mathrm{ac}$ levels were not reduced in $g c n 5 \Delta$ $d c r 1 \Delta$ cells as compared with $d c r 1 \Delta$ cells (Fig. 4B). These results suggest that the effects on pericentric heterochromatin function in the absence of RNAi are unique to the Mst2 complex. Because the H3K14R mutation abolished Swi6 recruitment and silencing at pericentric regions (Mellone et al. 2003), an effect not duplicated by fully abrogating H3K14ac through enzymatic inactivation (Supplemental Fig. S7), we could not definitively establish whether H3K14 is the sole target of the Mst2 complex at pericentric regions. Thus, although our results suggest that it is highly likely that Mst2 directly acetylates H3K14 at pericentric regions in the absence of RNAi, it is possible that Mst2 acetylates other substrates that indirectly affect H3K14ac levels. Taken together, these results demonstrate that RNAi-mediated heterochromatin assembly excludes access of the Mst2 histone acetyltransferase complex to prevent $\mathrm{H} 3 \mathrm{~K} 14 \mathrm{ac}$ at pericentric regions.

Consistent with the fact that $\mathrm{H} 3 \mathrm{~K} 14 \mathrm{ac}$ levels are positively correlated with transcription rates (Pokholok et al. 2005; Wang et al. 2008), we found that both Pol II protein levels at pericentric regions and pericentric transcript levels were high in $d c r 1 \Delta$ cells, but were significantly reduced in mst2 $2 \Delta$ cr $1 \Delta$ cells (Fig. 4C,D; Supplemental Fig. $\mathrm{S} 8 \mathrm{~A})$. Increased gene transcription, such as that in $d c r 1 \Delta$ cells, might result in nucleosome displacement as well as altered nucleosome modifications (Dion et al. 2007; Li et al. 2007). This could, in turn, affect the localization of the CLRC complex, which binds to pre-existing H3K9me to facilitate heterochromatin spreading and maintenance

(Zhang et al. 2008). Indeed, the loss of Dicer leads to the delocalization of CLRC components Clr4 and Raf2 at pericentric regions (Fig. 4E; Supplemental Fig. S8B). In contrast, in mst2d dcr1s cells, Pol II level is reduced and CLRC localization is restored (Fig. 4C,E; Supplemental Fig. S8B). Transcription of DNA repeats by Pol II during the $S$ phase is required for the generation of siRNAs through the RNAi pathway, which targets histone-modifying activities to heterochromatic loci (Cam et al. 2009), and mutations in Pol II subunits result in defective heterochromatin assembly (Djupedal et al. 2005; Kato et al. 2005). However, mutations in the Mst2 complex have little effect on the stability of pericentric heterochromatin (Figs. 1, 2; Gomez et al. 2005), and the Mst 2 complex does not show cell cycle-dependent localization at pericentric regions (Supplemental Fig. S9). Thus, it is unlikely that Mst2 affects S-phase-specific transcription of pericentric repeats in the presence of RNAi.

A recent large-scale epistasis analysis showed that RNAi mutants exhibit positive genetic interactions with mutations of the Mst2 complex, the RNA polymerase Mediator complex, the general transcription machinery, and a JmjC domain protein, Epe1 (Roguev et al. 2008). Epe1 promotes the localization of Pol II to heterochromatin (Zofall and Grewal 2006), and epe $1 \Delta d c r 1 \Delta$ cells can also maintain pericentric heterochromatin structures (Trewick et al. 2007). This suggests that limiting the access of Pol II to pericentric repeats by independent means can alleviate defects in heterochromatin maintenance associated with the loss of RNAi.

To further test this suggestion, we examined the effect of deleting a Mediator complex component (pmc2s) on RNAi-mediated heterochromatin maintenance. We found that pmc $2 \Delta d c r 1 \Delta$ cells also maintain silencing of otr::ura $4^{+}$ to some extent (Fig. 5A). Furthermore, there are higher levels of heterochromatin marks such as $\mathrm{H} 3 \mathrm{~K} 9$ me and Swi6 proteins in pmc2s dcr1s cells than in $d c r 1 \Delta$ cells (Fig. 5B). In addition, pmc $2 \Delta d c r 1 \Delta$ cells partially rescue TBZ sensitivity associated with $d c r 1 \Delta$ (Fig. 5A). However, pericentric heterochromatin in pmc2 $2 \Delta d c r 1 \Delta$ cells is not as stable as in mst2 $\Delta$ dcr1s cells, and silencing gradually deteriorates during passages (S Jia, unpubl.).

We thus further examined the stability of the silenced states with an otr::ade ${ }^{+}$reporter gene, the silencing of which results in the formation of red colonies when cells are grown in low-adenine medium. The culture of freshly made pmc2s dcr1s cells contains a mixture of red and white colony-forming cells, indicating silenced and expressed states of ade $6^{+}$, respectively (Fig. 5C), demonstrating that pmc2s $d c r 1 \Delta$ cells can maintain heterochromatin structures. While further growth of red cells gives rise to both red and white cells, white cells cannot revert to red cells (S Jia, unpubl.), indicating that these cells maintain heterochromatin only partially, but cannot re-establish heterochromatin once lost. As a result, the proportion of pmc $2 \Delta d c r 1 \Delta$ cells that retains silencing is gradually reduced upon continued growth (S Jia, unpubl.). The effect of pmc2 $\Delta$ dcr1s on transcription at pericentric regions is weaker than that of mst2s dcr1s (Supplemental Fig. S10). Thus, it seems that the ability to maintain heterochromatin in the absence of RNAi is correlated with the extent of reduction in RNA Pol II 
Reddy et al.

A

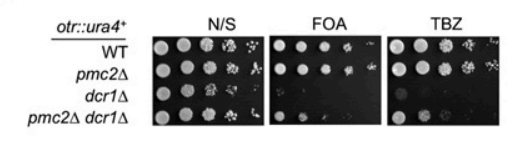

C

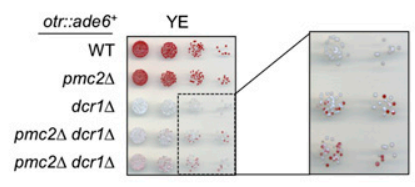

B

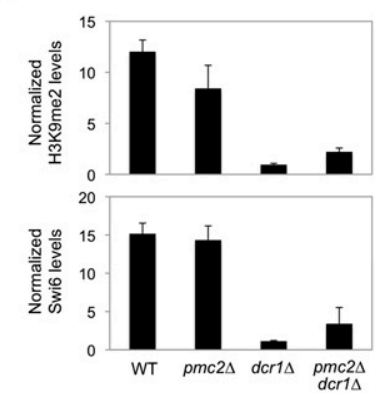

D

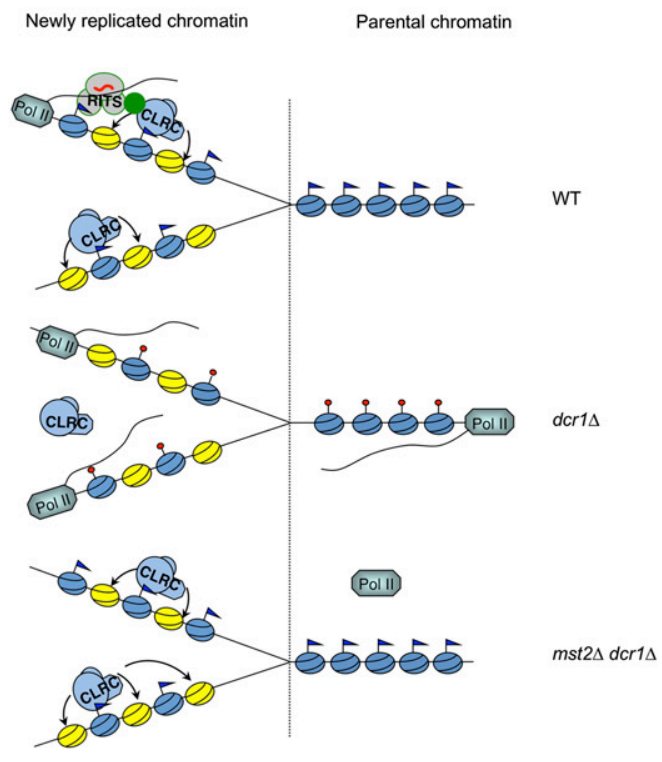

Figure 5. Loss of RNA Pol II Mediator component Pmc2 bypasses the requirement of the RNAi machinery for heterochromatin assembly. (A) Tenfold serial dilutions were performed to measure the expression of otr::ura $4^{+}$and the sensitivity to TBZ. The pmc $2 \Delta$ dcris otr::ura $4^{+}$strain was generated by crossing a pmc2 $\Delta d c r 1 \Delta$ strain with an otr::ura4 ${ }^{+}$strain, and freshly germinated cells were used for serial dilution assays and ChIP analysis. (B) ChIP analysis of H3K9me2 and Swi6 levels at otr::ura4 ${ }^{+}$. (C) The pmc2s dcr1s otr::adeb ${ }^{+}$strain was

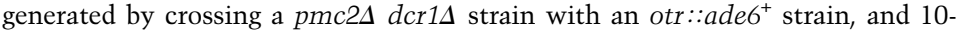
fold serial dilutions of freshly germinated yeast cells were spotted onto lowadenine media (YE) to measure the expression of otr::ade $6^{+}$. (D) A model of heterochromatin maintenance in the absence of RNAi-mediated heterochromatin establishment. (Top) In wild-type cells, S-phase-specific transcription results in the production of siRNAs and continued recruitment of CLRC to pericentric regions to reinitiate heterochromatin assembly. In addition, pre-existing $\mathrm{H} 3 \mathrm{~K} 9 \mathrm{me}$ (blue flags) is distributed randomly into newly replicated DNA during DNA replication, which helps recruit CLRC to methylate newly deposited histones, resulting in inheritance of this epigenetic state. (Middle) In RNAi mutants, higher levels of transcription result in the incorporation of histones without H3K9me or with other modifications (red lollipops). Without RNAi-mediated reinitiation, the absence of "seed" H3K9me after DNA replication to recruit CLRC prevents restoration of $\mathrm{H} 3 \mathrm{~K} 9 \mathrm{me}$ patterns, and the heterochromatin state is not maintained. (Bottom) In the absence of RNAi, reducing transcription slows nucleosome turnover, allowing maintenance of $\mathrm{H} 3 \mathrm{~K} 9$ me patterns after DNA replication without continued reinitiation.

of pericentric heterochromatin is critical for establishing functional centromeres (Malik and Henikoff 2009; Buscaino et al. 2010). Despite their essential functions, however, neither centromeric nor pericentric DNA sequences are evolutionarily conserved (Malik and Henikoff 2009). In multicellular eukaryotes, it has been suggested that meiotic drive, in which asymmetry in female meiosis leads to the retention of only one of four meiotic products, promotes rapid coevolution of centromeric and pericentric DNA sequences (Malik and Henikoff 2009). Fission yeast, which engages in symmetrical meiosis, nevertheless has significant differences of centromeric and pericentric DNA organization even among different isolates (Steiner et al. 1993). Thus, there must be additional mechanisms responsible for the accelerated evolution of centromeric and pericentric sequences. The highly homogeneous and tandemly arranged repetitive DNA elements common to pericentric regions are best explained by extensive recombination (Peng and Karpen 2008; Talbert and Henikoff 2010). However, these repeats are preferred targets for the assembly of heterochromatin, which strongly represses recombination (Peng and Karpen 2008; Ellermeier et al. 2010). Our data suggest that competing forces, such as the Mst2 complex and the RNAi machinery, regulate heterochromatin stability and thereby the efficiency of meiotic recombination at pericentric regions, which might permit fine-tuned evolution of pericentric sequences. Histone acetyltransferases in the MYST family are highly conserved across species, making it plausible that similar mechanisms regulate the evolution of pericentric heterochromatin in multicellular eukaryotes.

\section{Materials and methods}

\section{Fission yeast strains and genetic analyses}

Yeast strains containing deletions of Mst2 complex components ( $m s t 2 \Delta, n t o 1 \Delta$, and $p t f 2 \Delta)$ and epitope-tagged versions of proteins (Nto1-myc and Raf2-Flag) were constructed using a PCR-based module method (Bähler et al. 1998). Strains containing deletions of RNAi or heterochromatin components (arb1s, arb2 $\Delta$,

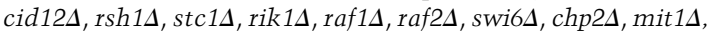
sir2s, and pmc2s) were purchased from Bioneer, verified via PCR, and backcrossed. Genetic crosses were used to construct all other strains. For serial dilution plating assays, 10-fold dilutions of a log-phase culture were plated on the indicated medium and grown for $3 \mathrm{~d}$ at $30^{\circ} \mathrm{C}$. The loss rate of minichromosome Ch16 was assayed as described previously (Hou et al. 2010). Meiotic recombination assays were performed as described previously (Ellermeier et al. 2010). Tetrad analysis was used to measure the recombination frequency. The generation of DNA DSBs during meiosis was analyzed by Southern blot hybridization as described previously (Ellermeier et al. 2010). transcription. Altogether, these results support our conclusion that reduced levels of RNA Pol II and transcription are critical to maintaining heterochromatin in the absence of RNAi (Fig. 5D).

Proper kinetochore assembly at centromeres is essential for accurate chromosome segregation (Allshire and Karpen 2008; Malik and Henikoff 2009), and the integrity

\section{Chromatin immunoprecipitation (ChIP)}

ChIP analysis was performed as described previously (Hou et al. 2010). Immunoprecipitation was performed with H3K9me2 (Abcam), Swi6, H3K14ac (Millipore), Pol II (8WG16, Covance), myc (Covance), or Flag (Sigma) antibodies. DNA isolated from ChIP or whole-cell extract was quantitatively analyzed by multiplex PCR with one primer pair amplifying different-sized PCR fragments from otr::ura $4^{+}$and the control ura4DS/E 
minigene. The ratios of signal intensities were used to calculate relative fold enrichment. Three biological repeats were performed for each ChIP experiment, and error bars represent standard deviation unless otherwise noted. Representative gels are shown.

\section{RNA analyses}

Total cellular RNA isolation, semiquantitative RT-PCR, and quantification with real-time RT-PCR were performed as described previously (Hou et al. 2010). Microarray analyses were performed as described previously (Lyne et al. 2003). The gene expression profile of $d c r 1 \Delta$ (Hansen et al. 2005) was also included for comparison. Northern blot of siRNAs was performed as described previously (Partridge et al. 2007).

\section{Acknowledgments}

We thank M. Borok and C. Santander for technical assistance; S. Kallgren for help with manuscript preparation; S. Grewal, R. Allshire, K. Ohta, H. Wang, K. Gull, and J. Partridge for yeast strains, reagents, and protocols; and J. Manley, D. Kalderon, and N. Phadnis for critical reading of the manuscript. This work was supported by National Institutes of Health grants R01-GM085145 (to S.J.) and R01-GM032194 (to G.R.S.), and a Cancer Research UK grant (to J.B.). B.D.R is supported by NIH training grant T32GM008798.

\section{References}

Allshire RC, Karpen GH. 2008. Epigenetic regulation of centromeric chromatin: Old dogs, new tricks? Nat Rev Genet 9: 923-937.

Allshire RC, Nimmo ER, Ekwall K, Javerzat JP, Cranston G. 1995. Mutations derepressing silent centromeric domains in fission yeast disrupt chromosome segregation. Genes Dev 9: 218-233.

Bähler J, Wu JQ, Longtine MS, Shah NG, McKenzie A III, Steever AB, Wach A, Philippsen P, Pringle JR. 1998. Heterologous modules for efficient and versatile PCR-based gene targeting in Schizosaccharomyces pombe. Yeast 14: 943-951.

Bayne EH, White SA, Kagansky A, Bijos DA, Sanchez-Pulido L, Hoe KL, Kim DU, Park HO, Ponting CP, Rappsilber J, et al. 2010. Stc1: A critical link between RNAi and chromatin modification required for heterochromatin integrity. Cell 140: 666-677.

Buhler M, Moazed D. 2007. Transcription and RNAi in heterochromatic gene silencing. Nat Struct Mol Biol 14: 1041-1048.

Buscaino A, Allshire R, Pidoux A. 2010. Building centromeres: Home sweet home or a nomadic existence? Curr Opin Genet Dev 20: 118-126.

Cam HP, Chen ES, Grewal SI. 2009. Transcriptional scaffolds for heterochromatin assembly. Cell 136: 610-614.

Dion MF, Kaplan T, Kim M, Buratowski S, Friedman N, Rando OJ. 2007. Dynamics of replication-independent histone turnover in budding yeast. Science 315: 1405-1408.

Djupedal I, Portoso M, Spahr H, Bonilla C, Gustafsson CM, Allshire RC, Ekwall K. 2005. RNA Pol II subunit Rpb7 promotes centromeric transcription and RNAi-directed chromatin silencing. Genes Dev 19: 2301-2306.

Ekwall K, Olsson T, Turner BM, Cranston G, Allshire RC. 1997. Transient inhibition of histone deacetylation alters the structural and functional imprint at fission yeast centromeres. Cell 91: 1021-1032.

Ellermeier C, Higuchi EC, Phadnis N, Holm L, Geelhood JL, Thon G, Smith GR. 2010. RNAi and heterochromatin repress centromeric meiotic recombination. Proc Nat1 Acad Sci 107: 8701-8705.

Gomez EB, Espinosa JM, Forsburg SL. 2005. Schizosaccharomyces pombe $\mathrm{mst}^{+}$encodes a MYST family histone acetyltransferase that negatively regulates telomere silencing. Mol Cell Biol 25: 8887-8903.

Grewal SI, Jia S. 2007. Heterochromatin revisited. Nat Rev Genet 8: 35-46.

Hall IM, Shankaranarayana GD, Noma K, Ayoub N, Cohen A, Grewal SI. 2002. Establishment and maintenance of a heterochromatin domain. Science 297: 2232-2237.

Hall IM, Noma K, Grewal SI. 2003. RNA interference machinery regulates chromosome dynamics during mitosis and meiosis in fission yeast. Proc Natl Acad Sci 100: 193-198.

Hansen KR, Burns G, Mata J, Volpe TA, Martienssen RA, Bahler J, Thon G. 2005. Global effects on gene expression in fission yeast by silencing and RNA interference machineries. Mol Cell Biol 25: 590-601.
Hou H, Wang Y, Kallgren SP, Thompson J, Yates JR III, Jia S. 2010. Histone variant H2A.Z regulates centromere silencing and chromosome segregation in fission yeast. J Biol Chem 285: 1909-1918.

Jia S, Noma K, Grewal SI. 2004. RNAi-independent heterochromatin nucleation by the stress-activated ATF/CREB family proteins. Science 304: 1971-1976.

Kato H, Goto DB, Martienssen RA, Urano T, Furukawa K, Murakami Y. 2005. RNA polymerase II is required for RNAi-dependent heterochromatin assembly. Science 309: 467-469.

Li B, Carey M, Workman JL. 2007. The role of chromatin during transcription. Cell 128: 707-719.

Lyne R, Burns G, Mata J, Penkett CJ, Rustici G, Chen D, Langford C, Vetrie D, Bahler J. 2003. Whole-genome microarrays of fission yeast: Characteristics, accuracy, reproducibility, and processing of array data. BMC Genomics 4: 27 . doi: 10.1186/1471-2164-4-27.

Malik HS, Henikoff S. 2009. Major evolutionary transitions in centromere complexity. Cell 138: 1067-1082.

Matzke MA, Birchler JA. 2005. RNAi-mediated pathways in the nucleus. Nat Rev Genet 6: 24-35.

Mellone BG, Ball L, Suka N, Grunstein MR, Partridge JF, Allshire RC. 2003. Centromere silencing and function in fission yeast is governed by the amino terminus of histone H3. Curr Biol 13: 1748-1757.

Motamedi MR, Hong EJ, Li X, Gerber S, Denison C, Gygi S, Moazed D. 2008. HP1 proteins form distinct complexes and mediate heterochromatic gene silencing by nonoverlapping mechanisms. Mol Cell 32: 778-790.

Nugent RL, Johnsson A, Fleharty B, Gogol M, Xue-Franzén Y, Seidel C, Wright AP, Forsburg SL. 2010. Expression profiling of S. pombe acetyltransferase mutants identifies redundant pathways of gene regulation. BMC Genomics 11: 59. doi: 10.1186/1471-2164-11-59.

Partridge JF, DeBeauchamp JL, Kosinski AM, Ulrich DL, Hadler MJ, Noffsinger VJ. 2007. Functional separation of the requirements for establishment and maintenance of centromeric heterochromatin. Mol Cell 26: 593-602.

Peng JC, Karpen GH. 2008. Epigenetic regulation of heterochromatic DNA stability. Curr Opin Genet Dev 18: 204-211.

Pokholok DK, Harbison CT, Levine S, Cole M, Hannett NM, Lee TI, Bell GW, Walker K, Rolfe PA, Herbolsheimer E, et al. 2005. Genome-wide map of nucleosome acetylation and methylation in yeast. Cell 122: 517-527.

Roguev A, Bandyopadhyay S, Zofall M, Zhang K, Fischer T, Collins SR, Qu H, Shales M, Park HO, Hayles J, et al. 2008. Conservation and rewiring of functional modules revealed by an epistasis map in fission yeast. Science 322: 405-410.

Sadaie M, Iida T, Urano T, Nakayama J. 2004. A chromodomain protein, $\mathrm{Chp1}$, is required for the establishment of heterochromatin in fission yeast. EMBO I 23: 3825-3835.

Steiner NC, Hahnenberger KM, Clarke L. 1993. Centromeres of the fission yeast Schizosaccharomyces pombe are highly variable genetic loci. Mol Cell Biol 13: 4578-4587.

Sugiyama T, Cam HP, Sugiyama R, Noma K, Zofall M, Kobayashi R, Grewal SI. 2007. SHREC, an effector complex for heterochromatic transcriptional silencing. Cell 128: 491-504.

Talbert PB, Henikoff S. 2010. Centromeres convert but don't cross. PLoS Biol 8: e1000326. doi: 10.1371/journal.pbio.1000326.

Trewick SC, Minc E, Antonelli R, Urano T, Allshire RC. 2007. The JmjC domain protein Epe1 prevents unregulated assembly and disassembly of heterochromatin. EMBO $J$ 26: 4670-4682.

Volpe T, Schramke V, Hamilton GL, White SA, Teng G, Martienssen RA, Allshire RC. 2003. RNA interference is required for normal centromere function in fission yeast. Chromosome Res 11: 137-146.

Wang Z, Zang C, Rosenfeld JA, Schones DE, Barski A, Cuddapah S, Cui K, Roh TY, Peng W, Zhang MQ, et al. 2008. Combinatorial patterns of histone acetylations and methylations in the human genome. Nat Genet 40: 897-903.

Zhang K, Mosch K, Fischle W, Grewal SI. 2008. Roles of the Clr4 methyltransferase complex in nucleation, spreading and maintenance of heterochromatin. Nat Struct Mol Biol 15: 381-388.

Zofall M, Grewal SI. 2006. Swi6/HP1 recruits a JmjC domain protein to facilitate transcription of heterochromatic repeats. Mol Cell 22: 681692. 


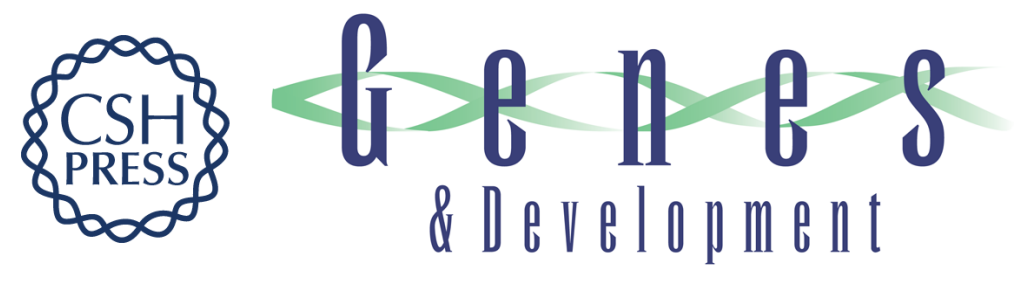

\section{Elimination of a specific histone H3K14 acetyltransferase complex bypasses the RNAi pathway to regulate pericentric heterochromatin functions}

Bharat D. Reddy, Yu Wang, Lifang Niu, et al.

Genes Dev. 2011, 25:

Access the most recent version at doi:10.1101/gad.1993611

Supplemental http://genesdev.cshlp.org/content/suppl/2011/02/02/25.3.214.DC1

Material

References This article cites 39 articles, 14 of which can be accessed free at: http://genesdev.cshlp.org/content/25/3/214.full.html\#ref-list-1

License

Email Alerting Receive free email alerts when new articles cite this article - sign up in the box at the top Service right corner of the article or click here.

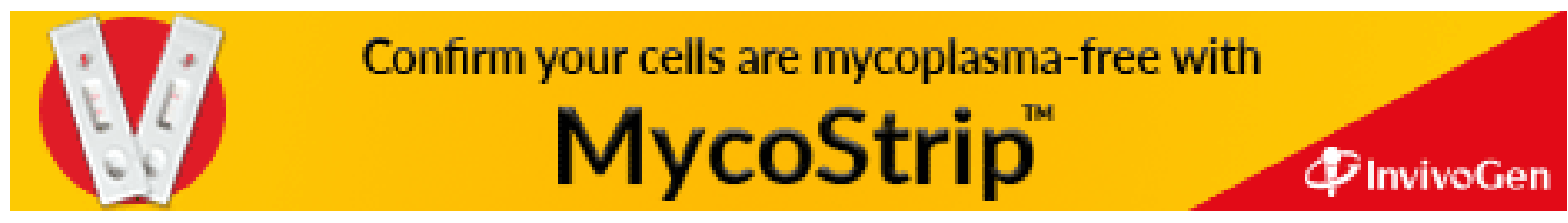

